\title{
Convergence and Extended Linear Generating Function for Generalized Hypergeometric Function
}

\author{
N. Srimannarayana, B. Satyanarayana, D. Ramesh
}

\begin{abstract}
The subject of Special functions has a lot importance during the last few decades. The intend of this work is to test the convergence and to introduce the extended linear generating relation for the generalized hypergeometric function. The result is followed by its applications to the classical polynomials.
\end{abstract}

Index Terms: Generalized Hypergeometric polynomial, Hypergeometric polynomials modified Jacobi polynomial, Laguerre polynomial.

\section{INTRODUCTION}

Many of the special functions like Laguerre, Hermite, Legendre and Konhauser polynomials has several applications in mathematical physics and statistics. Few decades onwards many researchers gave their generalizations, extensions and related properties like generating functions, extended generating functions, recurrence relations and solved some of the integrals using these functions. Previously in the paper [10], we defined a class of generalized hypergeometric function $B_{n}^{(\alpha, \beta)}(x, y, w)$ defined as follows:

$$
\begin{aligned}
B_{n}^{(\alpha, \beta)}(x, y, w) & =\frac{\Gamma(n+\alpha+1) \Gamma(n+\beta+1)}{n !} \\
& \times \sum_{r=0}^{n} \frac{(-1)^{r} y^{[r w]} J_{n-r}^{(\alpha)}(x, w)}{r ! \Gamma(n+\alpha-r+1) \Gamma(\mathrm{r}+\beta+1)}
\end{aligned}
$$

where $J_{n}^{(\alpha)}(x, w)$ is modified Jacobi polynomial which has been defined by using difference operator technique[3] (see Parihar and Patel [7] and also see Lahiri and Satyanarayana [4]-[6]). Also it has been derived the hypergeometric representation of (1) as see[10])

$$
\begin{aligned}
B_{n}^{(\alpha, \beta)}(x, y, w) & =\frac{(1+\alpha)_{n}(1+\beta)_{n}}{(n !)^{2}} \\
& \times \sum_{r=0}^{n} \sum_{s=0}^{n-r} \frac{(-n)_{r+s}\left(-\frac{y}{w}\right)_{r}\left(\frac{x}{w}\right)_{s}(-w)^{r}(w)^{s}}{r ! s !(1+\alpha)_{s}(1+\beta)_{r}}
\end{aligned}
$$

$$
=\frac{(1+\alpha)_{n}(1+\beta)_{n}}{(n !)^{2}} F_{-: 1 ; 1}^{1: 1 ; 1}\left[\begin{array}{l}
-n:-\frac{y}{w}, \frac{x}{w} ;-w, w \\
-: 1+\beta ; 1+\alpha ;
\end{array}\right]
$$

where $F_{q: s, v}^{p: r, u}$ is a double hypergeometric function (see [1]). By considering the limit $w \rightarrow 0 ; \beta=0, y=0$ and $w \rightarrow 0 ; \alpha=0, x=0$ and $w \rightarrow 0$ in (2), it leads to the following special cases

$$
\begin{aligned}
L_{n}^{(\alpha, \beta)}(x, y)= & \frac{(1+\alpha)_{n}(1+\beta)_{n}}{(n !)^{2}} \\
& \times \sum_{r=0}^{n} \sum_{s=0}^{n-r} \frac{(-n)_{r+s}(y)^{r}(x)^{s}}{r ! s !(1+\alpha)_{s}(1+\beta)_{r}}
\end{aligned}
$$

where $L_{n}^{(\alpha, \beta)}(x, y)$ is Laguerre polynomial of two variables defined by Ragab, S.F. [8].

By writing $\beta=0, y=0$ and taking $w \rightarrow 0$ in (2), it reduces to

$$
\operatorname{Lt}_{w \rightarrow 0} B_{n}^{(\alpha, 0)}(x, 0, w)=L_{n}^{\alpha}(x)
$$

where $L_{n}^{\alpha}(x)$ is Laguerre polynomial, Rainville, E.D.[9]. By writing $\alpha=0, x=0$ and taking $w \rightarrow 0$ in (2), it reduces to

$$
\underset{w \rightarrow 0}{L t} B_{n}^{(0, \beta)}(0, y, w)=L_{n}^{\beta}(y)
$$

where $L_{n}^{\beta}(y)$ is Laguerre polynomial, Rainville, E.D.[9]. The generating function of the form given by A.K.Agarwal and H.L.Manocha[1] is useful for obtaining the bilateral and trilateral generating relations for the generalized hypergeometric functions and is as

$$
\sum_{n=0}^{\infty} A_{m, n} \mathrm{~S}_{m+n}(x) \mathrm{t}^{n}=\frac{f(x, t)}{[g(x, t)]^{m}} S_{m}(h(x, t))
$$

Revised Version Manuscript Received on 16 September, 2019.

N.Srimannarayana, Department of Mathematics, Koneru Lakshmaiah Education Foundation, Vaddeswaram, Guntur, Andhra Pradesh, India-522502.

B. Satyanarayana, Department of Mathematics, Acharya Nagarjuna University, Nagarjuna Nagar, Andhra Pradesh, India-522510.

D. Ramesh, Department of Mathematics, Koneru Lakshmaiah Education Foundation, Vaddeswaram, Guntur, Andhra Pradesh, India-522502. 


\section{CONVERGENCE FOR $B_{n}^{(\alpha, \beta)}(x, y, w)$}

On account of (1), it can be obtained

$$
B_{n}^{(\alpha, \beta)}(x, y, w)=\sum_{r=0}^{n} \frac{(-n)_{r}\left(-\frac{y}{w}\right)_{r}(-w)^{r}}{r !(1+\beta)_{r}} A_{n, r}
$$

where

$$
A_{n, r}=\frac{(1+\alpha)_{n}(1+\beta)_{n}}{(n !)^{2}}{ }_{2} F_{1}\left[\begin{array}{c}
-n+r, \frac{x}{w} ; w \\
1+\alpha ;
\end{array}\right]
$$

Here $A_{n, r}$ is a polynomial and hence convergent.

$$
\begin{aligned}
& \text { Now, } \lim _{r \rightarrow \infty}\left|\begin{array}{c}
\frac{(-n)_{r+1}\left(-\frac{y}{w}\right)_{r+1}(-w)^{r+1}}{(r+1) !(1+\beta)_{r+1}} \\
\times \frac{r !(1+\beta)_{r} A_{n, r+1}}{(-n)_{r}\left(-\frac{y}{w}\right)_{r}(-w)^{r} A_{n, r}}
\end{array}\right| \\
& =\lim _{r \rightarrow \infty}\left|\frac{(-n+r)\left(-\frac{y}{w}+r\right) w}{(r+1)(1+\beta+r)}\right| \lim _{r \rightarrow \infty}\left|\frac{A_{n, r+1}}{A_{n, r}}\right| \\
& =|w s|
\end{aligned}
$$

where $s=\lim _{r \rightarrow \infty}\left|\frac{A_{n, r+1}}{A_{n, r}}\right|$. Thus, it can be concluded that the given function is convergent if $|w s|>1$ i.e., $|w|>\frac{1}{|s|}$ and divergent if $|w s|<1$ i.e., $|w|<\frac{1}{|s|}$. Moreover, if $|w s|=1$, then it can be proceeded as follows:

Let $\delta=\frac{1}{2} \operatorname{Re}\left(\frac{y}{w}+1+\beta+n\right)>0$ and by comparing the terms of the series.

$B_{n}^{(\alpha, \beta)}(x, y, w)$

$$
=A_{n, 0}+\sum_{r=1}^{n} \frac{(-n)_{r}\left(-\frac{y}{w}\right)_{r}(-w)^{r}}{r !(1+\beta)_{r}} A_{n, r}
$$

with the corresponding terms of the series $\sum_{r=1}^{\infty} \frac{1}{r^{1+\delta}}$, which is known to be convergent, the following can be obtained $\lim _{r \rightarrow \infty}\left|\frac{r^{1+\delta}(-n)_{r}\left(-\frac{y}{w}\right)_{r}}{r !(1+\beta)_{r} s^{r}} A_{n, r}\right|$

$=\frac{\rho}{\Gamma(-n)} \cdot \frac{1}{\Gamma\left(-\frac{y}{w}\right)} \cdot \frac{\Gamma(1+\beta)}{1} \cdot \lim _{r \rightarrow \infty}\left|\frac{1}{s^{r} r^{1+\beta+\frac{y}{w}+n-\delta}}\right|=0$ where $\lim _{r \rightarrow \infty}\left|A_{n, r}\right|=\rho$, is finite. Thus,

$\operatorname{Re}\left(\frac{y}{w}+1+\beta+n-\delta\right)=2 \delta-\delta=\delta>0$ and ' $s$ ' is finite. Hence, the function $B_{n}^{(\alpha, \beta)}(x, y, w)$ is absolutely convergent on $|w s|=1$ when $\operatorname{Re}\left(\frac{y}{w}+1+\beta+n\right)>0$. So, as the function (1) is convergent, one may get the different type of integrals, integral transforms like Laplace, Millen and Eugler-Beta for the same.

\section{EXTENDED LINEAR GENERATRING RELATION}

Now, we prove the following extended generating relation for the generalized hypergeometric function (1).

\section{Theorem 5 :}

$$
\begin{gathered}
\sum_{n=0}^{\infty}\left(\begin{array}{c}
m+n \\
n
\end{array}\right) \frac{B_{m+n}^{(\alpha, \beta)}(x, y, w) t^{n}(n+m) !}{(1+\alpha)_{(m+n)}(1+\beta)_{(m+n)}} \\
=\sum_{n+r=m}^{\infty}\left(\begin{array}{c}
n+r \\
m
\end{array}\right) \frac{t^{n+r-m}(-y / w)_{r} J_{n}^{\alpha}(x, w) \mathrm{w}^{r}}{\mathrm{r} !(1+\alpha)_{n}(1+\beta)_{r}} \\
\quad \times{ }_{1} F_{1}[n+r+1 ; n+r-m+1 ; t]
\end{gathered}
$$

Proof : The proof of (11) can be developed easily from (1) in the following way

Consider the double series

$$
\sum_{m=0}^{\infty} u^{m} \sum_{n=0}^{\infty}\left(\begin{array}{c}
m+n \\
n
\end{array}\right) \frac{B_{m+n}^{(\alpha, \beta)}(x, y, w) t^{n}(n+m) !}{(1+\alpha)_{m+n}(1+\beta)_{m+n}}
$$

and on replacing ' $n$ ' by '(n-m)' and using Satyanarayana and others[10, pp.263(3.2)], we obtain

$$
\begin{aligned}
=\sum_{n, r, v=0}^{\infty} & \frac{\left(-\frac{y}{w}\right)_{r} J_{n}^{\alpha}(x, w) w^{r}}{(1+\alpha)_{n}(1+\beta)_{r} r ! v !} \\
& \times \sum_{m=0}^{n+r+v} \frac{(n+r+v) ! \mathrm{u}^{m} t^{n+r+v-m}}{m !(n+r+v-m) !}
\end{aligned}
$$




$$
\begin{aligned}
& =\sum_{m=0}^{\infty} u^{m} \sum_{n+r+v \geq m} \frac{(n+r) !(n+r+1)_{v}}{(n+r-m+1)_{v}} \\
& \quad \times \frac{t^{n+r-m}\left(-\frac{y}{w}\right)_{r} J_{n}^{\alpha}(x, w) w^{r} t^{v}}{r ! v ! m !(n+r-m) !(1+\alpha)_{n}(1+\beta)_{r}} \\
& =\sum_{m=0}^{\infty} u^{m} \sum_{n+r=0}^{\infty} \frac{n+r_{C_{m}} t^{n+r-m}\left(-\frac{y}{w}\right)_{r} J_{n}^{\alpha}(x, w) w^{r}}{(1+\alpha)_{n}(1+\beta)_{r} r !} \\
& \times{ }_{1} F_{1}\left[\begin{array}{c}
n+r+1 ; \\
n+r-m+1 ;
\end{array}\right]
\end{aligned}
$$

\section{EXPERIMENTAL RESULTS}

Case 1 :- If $m=0$, the above result leads to

$$
\begin{aligned}
& \sum_{n=0}^{\infty} \frac{n ! B_{n}^{(\alpha, \beta)}(x, y, w) t^{n}}{(1+\alpha)_{n}(1+\beta)_{n}} \\
& =e^{t}{ }_{1} F_{1}\left(-\frac{y}{w} ; 1+\beta ; w t\right){ }_{1} F_{1}\left(\frac{x}{w} ; 1+\alpha ;-w t\right)
\end{aligned}
$$

which is the known result by Satyanarayana and others[10, pp.263(3.2)]

Case 2 :- If $m=0$ and by taking $w \rightarrow 0$ on both sides of the above result, we get the known result by S.K.Chatterjea[11]

$$
\begin{aligned}
& \sum_{n=0}^{\infty} \frac{n ! L_{n}^{(\alpha, \beta)}(x, y) t^{n}}{(1+\alpha)_{n}(1+\beta)_{n}} \\
& \quad=e^{t}{ }_{0} F_{1}(-; 1+\alpha ;-x t)_{0} F_{1}(-; 1+\beta ;-y t)
\end{aligned}
$$

\section{CONCLUSION}

This work has tested the union of the function (1) and obtained extended generating relation with application as special cases. By using the this extended linear generating relation, one may get the extended bilinear and bilateral generating associations through classical and generalized hypergeometric functions. By taking those bilateral generating functions, obtain very easily the integrals of the product of the functions which are useful in some of the engineering problems and also in mathematical physics.

\section{ACKNOWLEDGMENT}

The first author is thankful to the management of Koneru Lakshmaiah Education Foundation for their constant encouragement in preparation of this paper.

\section{REFERENCES}

1. A.K.Agarwal and H.L.Manocha, On some new generating functions, Indian J.Pure and Pure and Appl. Mathematics., Vol.13, No.11, 1369-1375, 1982.

2. H.M.Srivastava and P.W.Karlsson, Multiple Gaussian Hypergeometric Series, Halsted Press (Ellis Horwood Limited Chichester) John Wiley and Sons, New York, 1985
3. L.M.Milne-Thomson, The Calculus of Finite Differences, Macmillan, London, 1951.

4. M.Lahiri and B.Satyanarayana, A class of generalized hypergeometric functions defined by using a difference operator, Soochow J. Math.19 (1993), 163-171.

5. M.Lahiri and B.Satyanarayana, Certain bilateral generating relations for generalized Hypergeometric functions, Proceedings of Indian Academy of Science (Mathematical Sciences), Vol.105, No.3, 297-301, 1995.

6. M.Lahiri and B.Satyanarayana, Discrete and pseudo orthogonality for a class of Generalized hypergeometric functions, Tamkong Journal of Mathematics, Vol.28, No.4, 325-332, 1997.

7. C.L.Parihar and V.C.Patel, On modified Jacobi polynomials, Journal of Indian Acad.Math., Vol.1, 41-46, 1979.Rainville, E.D., Special Functions, The Macmillan Co. Inc., New York, 1960.

8. Ragab, S.F., On Laguerre polynomial of two variables, Bull.Cal.Math.Soc., Vol.83, 253-262, 1991.

9. Rainville, E.D., Special Functions, Macmillan, New York, 1960.

10. Satyanarayana B, Srimannarayana N and Pragathi Kumar Y, A class of generalized hypergeometric functions of two discrete variables, Analele Universittii Constantin Brancusi Din Targu Jiu, Seria Inginerie, Vol.3, 260-269, 2010.

11. S.K.Chatterjea, A note on Laguerre polynomials of two variables, Bull.Cal.Math.Soc. 82(1991), 263-266.

\section{AUTHORS PROFILE}

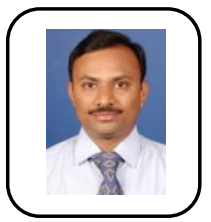

Dr.N.Srimannarayana working as Professor in the Department of Mathematics, Koneru Lakshmaiah Education Foundation, Vaddeswaram, Guntur, Andhra Pradesh, India-522502. He is having 22+ years of teaching experience in various Degree and Engineering Colleges. His research area of interest is Special Functions, Differential Equations and Fuzzy algebra . He is a life member in S.S.F.A and A.P.T.M.S

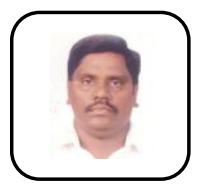

Dr.B.Satyanarayana working as Coordinator, Department of Mathematics and Chairman, P.G.-B.O.S in Mathematics, Acharya Nagarjuna University, Nagarjunanagar, Guntur(Dt.,), Andhra Pradesh, India-522510. Under his guidance 10 Ph.D.'s and 8 M.Phil's awarded. He published more than $82+$ research papers in reputed Natiional and International Journals. His area of research is Special Functions and Fuzzzy algebra.

Dr.D.Ramesh working as Assistant Professor in the Department of Mathematics, Koneru Lakshmaiah Education Foundation, Vaddeswaram, Guntur, Andhra Pradesh-522502, India. $\mathrm{He}$ is having 14+ years of teaching experience in various Degree, P.G. and Engineering Colleges. His research area of interest is Fuzzy algebra and Special Functions. He published more than 15 Research papers in reputed National and International Journals. He is a life member in A.P.T.M.S. 